\title{
Tailored plasma-density profiles for enhanced energy extraction in passive plasma beam dumps
}

DOI:

10.1088/1361-6587/ab4cfb

Document Version

Accepted author manuscript

Link to publication record in Manchester Research Explorer

\section{Citation for published version (APA):}

Jakobsson, O., Bonatto, A., Li, Y., Zhao, Y., Pizzato Nunes, R., Williamson, B., Xia, G., \& Tajima, T. (2019).

Tailored plasma-density profiles for enhanced energy extraction in passive plasma beam dumps. Plasma Physics and Controlled Fusion. https://doi.org/10.1088/1361-6587/ab4cfb

\section{Published in:}

Plasma Physics and Controlled Fusion

\section{Citing this paper}

Please note that where the full-text provided on Manchester Research Explorer is the Author Accepted Manuscript or Proof version this may differ from the final Published version. If citing, it is advised that you check and use the publisher's definitive version.

\section{General rights}

Copyright and moral rights for the publications made accessible in the Research Explorer are retained by the authors and/or other copyright owners and it is a condition of accessing publications that users recognise and abide by the legal requirements associated with these rights.

\section{Takedown policy}

If you believe that this document breaches copyright please refer to the University of Manchester's Takedown Procedures [http://man.ac.uk/04Y6Bo] or contact uml.scholarlycommunications@manchester.ac.uk providing relevant details, so we can investigate your claim.

\section{OPEN ACCESS}


ACCEPTED MANUSCRIPT

\title{
Tailored plasma-density profiles for enhanced energy extraction in passive plasma beam dumps
}

To cite this article before publication: Oscar Jakobsson et al 2019 Plasma Phys. Control. Fusion in press https://doi.org/10.1088/1361$\underline{6587 / a b 4 c f b}$

\author{
Manuscript version: Accepted Manuscript \\ Accepted Manuscript is "the version of the article accepted for publication including all changes made as a result of the peer review process, \\ and which may also include the addition to the article by IOP Publishing of a header, an article ID, a cover sheet and/or an 'Accepted \\ Manuscript' watermark, but excluding any other editing, typesetting or other changes made by IOP Publishing and/or its licensors" \\ This Accepted Manuscript is @ 2019 IOP Publishing Ltd.
}

During the embargo period (the 12 month period from the publication of the Version of Record of this article), the Accepted Manuscript is fully protected by copyright and cannot be reused or reposted elsewhere.

As the Version of Record of this article is going to be / has been published on a subscription basis, this Accepted Manuscript is available for reuse under a CC BY-NC-ND 3.0 licence after the 12 month embargo period.

After the embargo period, everyone is permitted to use copy and redistribute this article for non-commercial purposes only, provided that they adhere to all the terms of the licence https://creativecommons.org/licences/by-nc-nd/3.0

Although reasonable endeavours have been taken to obtain all necessary permissions from third parties to include their copyrighted content within this article, their full citation and copyright line may not be present in this Accepted Manuscript version. Before using any content from this article, please refer to the Version of Record on IOPscience once published for full citation and copyright details, as permissions will likely be required. All third party content is fully copyright protected, unless specifically stated otherwise in the figure caption in the Version of Record.

View the article online for updates and enhancements. 


\section{Tailored plasma-density profiles for enhanced energy extraction in passive plasma beam dumps}

O Jakobsson $^{1}$, A Bonatto ${ }^{1,2}, \mathbf{Y} \mathbf{L i}^{1,2}, \mathbf{Y}$ Zhao ${ }^{1,2}, \mathbf{R} \mathbf{P}$ Nunes $^{3}$, B Williamson ${ }^{1,2}$, G Xia ${ }^{1,2}$, T Tajima ${ }^{4}$

${ }^{1}$ School of Physics and Astronomy, University of Manchester, Oxford Road, Manchester M13 9PL, United Kingdom

${ }^{2}$ The Cockcroft Institute, Sci-Tech Daresbury, Daresbury, Warrington, WA4 4AD, United Kingdom

3 Departamento de Engenharia Elétrica, Escola de Engenharia, Universidade Federal do Rio Grande do Sul, Porto Alegre, Brazil

4 Department of Physics and Astronomy, University of California Irvine, California, US

E-mail: alexandre.bonatto@manchester.ac.uk

E-mail: guoxing.xia@manchester.ac.uk

Abstract. Plasma-based deceleration could greatly improve the overall compactness of accelerator facilities. In the so-called passive plasma beam dump, collective oscillations of plasma electrons are used to absorb kinetic energy of spent high-energy electron beams. Moreover, due to the high-amplitude decelerating gradient and the low-density plasma medium, deceleration is achieved in a compact and safer way, if compared to conventional beam dumps. Adoption of this novel decelerating scheme might be critical for facilities aiming for high-energy, high-repetition-rates, as well as for transportable applications built upon plasmabased accelerator technology. Howeverr, key issues such as, for example, particle re-acceleration, need to be addressed. In this work, tailored plasma-density profiles are used to shift the defocusing phase of the beam self-driven transverse wakefield towards re-accelerated beam particles, and transversely eject them. PIC simulations are used to evaluate the total beam energy, energy deposited in the plasma, and energy transversely ejected, for each of the investigated plasma density profiles. Moreover, the average energy per ejected particle is estimated for each case. In particular, it is shown that the energy of ejected particles is affected by the rate at which the plasma wavelength decreases, since it determines the time that particles experience re-acceleration before being defocused.

Keywords: plasma beam dump, beam energy loss, beam-driven wakefield, beam deceleration, EuPRAXIA, PWFA. 


\section{Introduction}

The development of compact, high-quality electron accelerators for the production of ultra-relativistic electron beams, with single-particle energies in the $\mathrm{GeV}$ range, is of great interest to many areas of contemporary science, industry, and medicine; promising to scale down the size requirements for high-quality $\mathrm{x}$-ray production, terahertz-radiation generation, and free-electron lasing. Plasma wakefield accelerators - whereby an ultra-relativistic particle beam or a high intensity laser pulse drives a plasma wakefield - are able to provide accelerating gradients up to hundreds of $\mathrm{GVm}^{-1}$, thousands of times higher than those in conventional radio-frequency cavities. Laser plasma accelerators (LPA) [1, 2], also known as laser wakefield accelerators (LWFA), can achieve sizeable energy gain in centimetre-long plasma cells using TW to PW laser pulses. Recent results include the guiding of an $850 \mathrm{TW}$ laser pulse through a 20 centimeter-long pre-formed plasma channel at the BELLA center, yielding a $5 \mathrm{pC}$ electron bunch at $7.8 \mathrm{GeV}$ [3]. In addition, serial acceleration of a single electron beam in two coupled LPA stages (LPA staging) was observed [4]. Since LPA staging can be used to overcome the laser depletion in laser plasma accelerators, this is an important milestone towards the design of future LPA-based linear colliders $[5,6]$.

Results also have been achieved using beam-driven plasma accelerators (PWFA) [7]. The experiment conducted at the Final Focus Test Beam (FFBT) facility at SLAC demonstrated that a fraction of a $42 \mathrm{GeV}$ electron beam had its energy doubled by its self-driven wakefield, which produced an accelerating gradient of approximately $52 \mathrm{GeV} / \mathrm{m}$ in an 85 centimeter-long plasma [8]. More recently, a $400 \mathrm{GeV}$ proton-bunch was used as a driver to demonstrate the trapping and acceleration of electrons in the AWAKE experiment at CERN [9]. Further advances are to be expected from the ongoing FLASHForward experiment at DESY [10], FACET-II at SLAC [11] and AWAKE run-2 [12].

As LPA and BPA technologies progress to higher repetition rates, bunch charges, and energies, the safe disposal of these beams will require correspondingly larger beam dumps if conventional technologies are applied. These beam dumps are usually meter-long, water-cooled, metal or graphite blocks $[13,14]$, attaining their stopping power from collisional, scattering and ionization mechanisms. As a result, conventional beam dumps suffer from nuclear radioactivation owing to the production of secondary particles, radiative emission from decelerating electrons, and photonuclear reactions, which must be carefully assessed and adequately shielded [15-17]. A complementary approach to reduce the power deposited in these conventional beam dumps, and hence the amount of induced radioactivation, is to use plasmas to extract energy from the beam prior to entering the beam dump [18]. These so-called plasma beam dumps take advantage of the initial accelerating and focusing phases of the longitudinal and transverse wakefields, respectively, excited by an electron bunch propagating in a plasma, to extract the bunch energy as it propagates. By tuning the plasma density against the longitudinal beam profile, decelerating wakefields with amplitudes in the $\mathrm{GV} / \mathrm{m}$ range can act across the beam; orders of magnitude larger than that achievable by collisional deceleration in dense matter [19]. Crucially, the relatively low-density of plasma in comparison to dense matter further leads to a significant reduction in radioactivations, since deceleration occurs through collective interaction rather than radiative-loss mechanisms [18]. Plasma beam dump studies fall into two configurations: passive, whereby an electron beam is decelerated by its self-driven wakefield; and active, in which a preceding beam or laser driver enhances the decelerating wakefield. Passive [18-21] and active [20, 22, 23] plasma beam dumps have been investigated theoretically. Proof-of-principle experiments have been conducted on LWFA electron beams, successfully demonstrating deceleration of electrons in a plasma due to self-driven collective wake excitations [21]. Furthermore, this form of self-driven beam deceleration has recently been used in proof-of-principle experiments $[24,25]$ to demonstrate its use as an energy "dechirper" for electron beams. Specifically, a negatively chirped longitudinal beam-energy profile - one which increases linearly from head to tail - was flattened by the beam self-driven wakefield. In addition, it has been demonstrated that an electron beam propagating outside a plasma column was attracted into the plasma, inside which beam deceleration was observed [26].

Previous results show that while in the active configuration more than $95 \%$ of the total beam energy is extracted [20], the passive case saturates at an energy extraction of $60 \%$ to $70 \%$ [18-20,27, 28], due to particle re-acceleration. However, the passive plasma beam

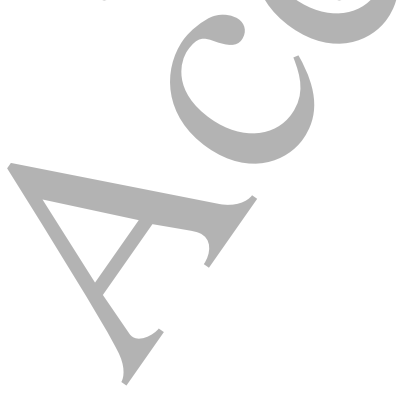


Tailored plasma-density profiles

dump scheme presents interesting advantages when compared to the active one. The first is associated with the energy involved in the dump system. In a passive beam dump, the decelerating wakefield is produced by the beam itself. In this way, beam energy is used to decelerate its own particles. In contrast, the active scheme requires an additional energy-source, the laser pulse, to decelerate the beam. The second is related to the complexity of the dump system. In the active system, further equipment is required in comparison to the passive configuration, in which just a plasma stage has to be implemented. The active configuration requires additional laser infrastructure and stringent synchronization in order to achieve an effective energy extraction. The aforementioned advantages motivate further investigation to improve the passive plasma beam dump before moving attention to the active case.

The goal of this work is to improve the total beamenergy extraction in the passive plasma beam dump scheme. By using tailored plasma-density profiles, particle re-acceleration is mitigated, and energy loss saturation is prevented. This allows for further beamenergy extraction, not achievable with a uniform plasma density. This work is organized as follows. In Section 2, beam deceleration in a passive plasma beam dump with uniform plasma-density profile, as well as the energy loss saturation caused by particle re-acceleration, are discussed. Section 3 investigates how well a distinct set of varying plasma-density schemes overcome the limitations identified in Section 2. Finally, in Section 4 the main contributions of this work are identified and summarized.

\section{Uniform plasma-density profile}

The linear fluid model of beam-driven plasma wakefield generation describes the response of a plasma to an ultra-relativistic electron beam, provided that the plasma density $n_{0}$ is much larger than the beam-density $n_{b}$, i.e., $n_{b} / n_{0} \ll 1$. However, studies show this model holds up to $n_{b} / n_{0} \simeq 10$ [29]. In this model, the longitudinal and transverse components of the beam self-driven wakefield have alternating decelerating-accelerating and focusingdefocusing phases, the wavelength of which are given by the plasma wavelength $\lambda \propto n_{0}^{-1 / 2}$. From now on, $\xi \equiv z-c t$ is defined as the local bunch coordinate, i.e., the co-moving coordinate. For a bi-Gaussian beam with longitudinal and transverse RMS widths $\sigma_{\xi}$ and $\sigma_{r}$, respectively, the longitudinal field rises from zero at its head up to its maximum value, $E_{\xi}^{\max }$, whose location depends on the beam length $L \equiv 4 \sigma_{\xi}$ ( $\sim 95 \%$ of beam particles) with respect to the plasma wavelength $\lambda$. For a short beam, defined by $k_{p} L \ll \pi$, where $k_{p}=$ $2 \pi / \lambda$ is the wavenumber, the wakefield grows nearly monotonically from beam head to tail. For a uniform plasma density profile, this results in a constant rate of beam-energy extraction with propagation distance, which can be calculated analytically for a given beam density profile [20], provided that all beam particles remain ultra-relativistic.

The behavior previously mentioned is illustrated in figure 1(a), which contains PIC simulation results for a bi-Gaussian electron beam undergoing a uniform passive plasma beam dump. For longer propagation distances, particles experiencing a higher amplitude wakefield will become non-relativistic, while particles at the head of the bunch still preserve their initial energy, as illustrated by figure 1(b). At this point, the rate of beam-energy extraction diminishes as nonrelativistic particles start falling behind the head of the bunch, into the accelerating phase of the longitudinal wakefield, where they are subsequently reaccelerated to relativistic energies. Figure 1(c) exhibits a secondary peak of beam particles, formed by reacceleration of decelerated particles. The continuing re-acceleration of these particles ends up balancing the energy extraction from the decelerating phase of the wakefield, and the net beam energy saturates as a result of this. Saturation length, the propagation distance after which saturation is observed, can be estimated $[18,19]$ and used to determine the minimum total beam energy achievable in a passive plasma beam dump with a uniform plasma-density profile [20]. Defining $U$ as the total beam energy at a given propagation distance, and $U_{0}$ as the initial total beam energy, previous PIC simulations $[18,20]$ show beams reaching saturation within $30 \%$ to $40 \%$ of their initial energy, i.e., with $0.3 \lesssim U / U_{0} \lesssim$ 0.4 , as further energy extraction is prevented by reacceleration. Addressing this issue, Wu et al [18] proposed to minimize re-acceleration by intersecting the plasma with thin foils, strategically placed to capture low-energy particles before significant reacceleration has occurred. This resulted in an energy loss of up to $90 \%$. It is however unclear how prolonged exposure to high-energy electron bunches and the conditions within the plasma would degrade these foils. As an alternative, Hanahoe et al [19] explored the use of varying plasma densities. Linear or quadratically increasing plasma-density profiles were shown to reduce re-acceleration by transversely defocusing particles falling behind the bunch, resulting in beam energies $U / U_{0} \sim 0.25$. Experimentally, the re-acceleration of decelerated particles was observed by Chou et al [21] in their aforementioned self-driven deceleration of LWFA beams. Furthermore, charge loss following the decelerating was attributed to scattering and defocusing of decelerated particles falling behind the bunch. 


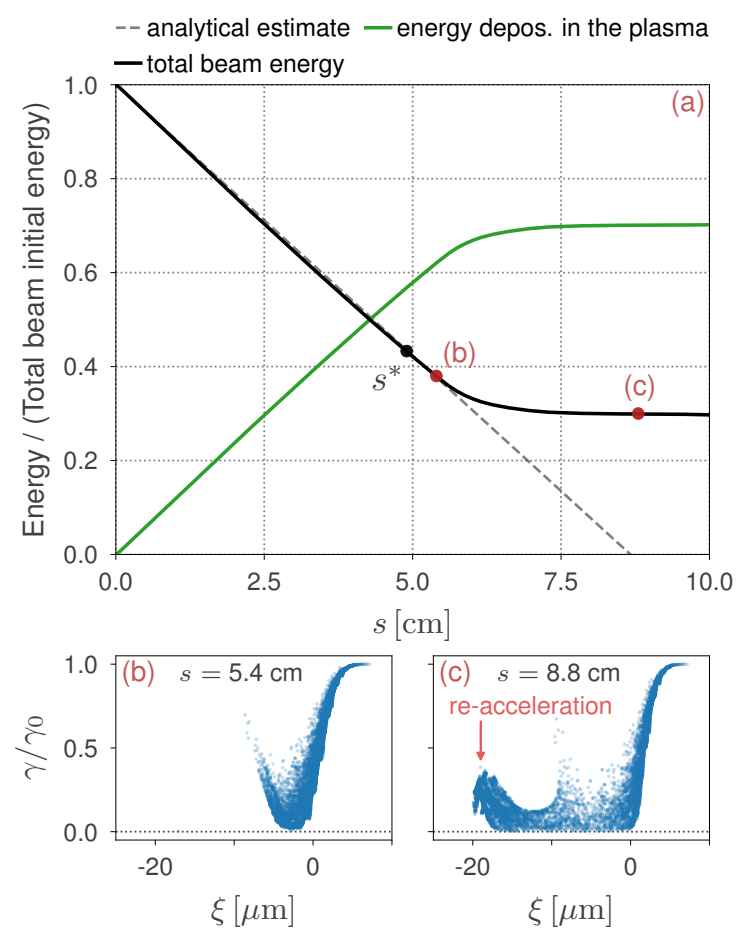

Figure 1. Total energy loss for a bi-Gaussian beam $\left(\sigma_{\xi}=\right.$ $2.0 \mu \mathrm{m}, \sigma_{r}=1.4 \mu \mathrm{m}$, and $Q=30 \mathrm{pC}$ ) propagating in a passive beam dump with uniform plasma density profile $\left(n_{0}=9.9 \times 10^{17} \mathrm{~cm}^{-3}, n_{b} / n_{0} \simeq 3\right.$, and $\left.k_{p} L=1.5\right)$. (a) PIC simulation results (black, solid line) shows beam-energy extraction at a constant rate, in agreement with the analytical estimate (grey, dashed line) for the total beam energy loss [20]. The agreement holds up to the saturation length, $s^{*} \simeq 5 \mathrm{~cm}$ $[18,19]$. The extracted energy is deposited in the plasma (green line). (b) After the saturation length $s^{*}$, at $s=5.4 \mathrm{~cm}$, particles experiencing higher amplitude wakefield are the first to become non-relativistic $\left(\gamma / \gamma_{0} \ll 1\right)$, while particles at the head of the bunch still preserve their initial energy. (c) At $s=8.8 \mathrm{~cm}$, there is an appreciable secondary peak, formed by re-acceleration of particles reaching the next accelerating phase of the wakefield behind the beam. This effect balances the energy extraction, saturating the net beam total energy loss.

In the passive beam dump examples above, the foremost region of the beam still maintains its initial energy, leading to a positive energy chirp with $100 \%$ energy spread. To address this issue, Bonatto et al [20] proposed the active beam dump scheme. By carefully positioning a laser driver ahead of the bunch, it was shown that the energy in the head could be significantly reduced, resulting in total energy losses up to $97 \%$. The need for laser-guiding plasma channels, precise timing and high-power lasers may, however, make this scheme difficult to implement at the present time.

In this paper, tailored plasma densities are further explored to defocus particles during re-acceleration. It is shown that up to $90 \%$ of the total beam energy can be extracted, with $80 \%$ being deposited into the plasma, and $10 \%$ carried away by the defocused particles. This is a significant improvement compared to the previous studies on the passive beam dump. In addition, the energy of these defocused particles is studied and it is shown that the rate at which the plasma wavelength decreases plays a crucial role in the amount of re-acceleration they experience before getting defocused.

\section{Tailored plasma-density profiles}

In the linear to the quasi-linear regime, the transverse and longitudinal wakefield components are out of phase by $\sim \pi / 2$, such that the maximum accelerating field behind the bunch occurs at the point where the transverse field changes from focusing to defocusing. As it happens, the re-acceleration of non-relativistic particles falling behind the bunch works such that the particles attain relativistic energies just before reaching the start of the defocusing region. As the bunch continues to lose particles, this leads to the formation of a secondary bunch of re-accelerating particles behind the head of the bunch, just in front of the defocusing region. Hence, by increasing the plasma density, the corresponding shortening of the plasma wavelength will move the defocusing region towards the head of the bunch, and over the re-accelerating particles. At this point, these particles are rapidly ejected in the transverse direction, thus avoiding the saturation of the beam-energy loss [19]. It has been shown that, even in a uniform density, saturation of beam-energy loss is eventually overcome by the defocusing of particles in the secondary bunch, the so-called re-acceleration peak, when its charge is large enough [30]. This however includes the ejection of particles with energies above the initial energy, with no major reduction in beam energy.

For the numerical simulations performed next, bunch parameters have been chosen to represent the proposed laser-plasma accelerator-EuPRAXIA [31]. Namely, a bunch with: an RMS length, $\sigma_{\xi}=2.0 \mu \mathrm{m}$; an RMS radius, $\sigma_{r}=1.4 \mu \mathrm{m}$; a bunch charge, $Q=$ $30 \mathrm{pC}$; a particle-energy, $E_{k 0}=1 \mathrm{GeV}$, corresponding to an initial relativistic factor of $\gamma_{0} \simeq 1960$. Particle energy has been lowered from the $5 \mathrm{GeV}$ aimed by the EuPRAXIA project to decrease the computational time and allow for a more comprehensive simulation study. However, if the propagation distance is properly scaled, results presented in this paper are applicable to the same beam with higher initial particle energies. Since the total beam energy loss is linear while the whole beam remains highly relativistic [18-20], if the same beam has particles with higher initial energy, it will have to propagate for a longer distance into the plasma in order to reach the saturation distance $s^{*}$. Other than that, in theory the presented results should remain valid. Simulations are performed using 
Tailored plasma-density profiles

the particle-in-cell (PIC) code FBPIC [32, 33], in a two-dimensional axisymmetric geometry to allow for accurate modeling of the transverse dynamics. FBPIC uses a spectral, quasi-cylindrical algorithm which avoids spurious numerical dispersion, including the zero-order numerical Cherenkov effect [34]. A simulation domain of $-2.5 \pi / k_{p} \leq \xi \leq 5 \sigma_{\xi}$ and $0<r \leq 20 \sigma_{r}$ was chosen to ensure that the reacceleration peak, formed by decelerated particles after phase slippage, as well as the defocused particles were properly evaluated. Longitudinal and transverse resolutions of $\sigma_{\xi} / 25$ and $\sigma_{r} / 25$, respectively, were adopted; the number of particles per cell was set to 4 , being 2 along each coordinate $z$ and $r$. Regarding the azimuthal Fourier decomposition [32] of the electromagnetic field components, a purely cylindrical mode $(m=0)$ was adopted.

In the next subsections, three distinct varying plasma-density schemes are presented. Extending the previous work by Hanahoe et al [19], particular focus is placed on the energy of the re-accelerated particles at ejection and the energy deposited in the plasma. In order to fit the bunch within the initial deceleration region, and allow room for the plasma wavelength to shrink, an initial plasma density $n_{0}=9.9 \times 10^{17} \mathrm{~cm}^{-3}$, such that $k_{p} L=1.5$, is chosen. This corresponds to a $n_{b} / n_{0} \simeq 3$, where the peak beam-density is given by

$$
n_{b}=\frac{Q / e}{(2 \pi)^{3 / 2} \sigma_{\xi} \sigma_{r}^{2}},
$$

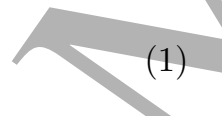

and $e$ is the electron charge. This choice of plasma density means that propagation is no longer in the linear regime, as defined by $n_{b} / n_{0} \ll 1$, but rather in the quasi-linear to non-linear regime. Even though the higher plasma density needed for the linear regime would increase the maximum decelerating field across the bunch, the shortened plasma wavelength would place the back-end of the bunch in the accelerating region from the start. This would accelerate electrons in this part of the bunch to above the initial energy, and defocusing of these particles would not be desirable. Despite the deviation from the linear regime, it is shown that the mechanisms of deceleration, reacceleration, and defocusing still behave similarly to the descriptions above.

In this work, $s=c t \simeq(\mathrm{d} z / \mathrm{d} t) t$ is assumed to be the beam propagation distance. Since both $s$ and $z$ are correlated, for the sake of simplicity, from now on $s$ will also be used to denote the spatial coordinate.

\subsection{Multi-staged uniform density scheme}

The idea of the first scheme is to let the beam propagate further after the beam energy loss saturation, allowing the formation of a re-acceleration peak. Then,

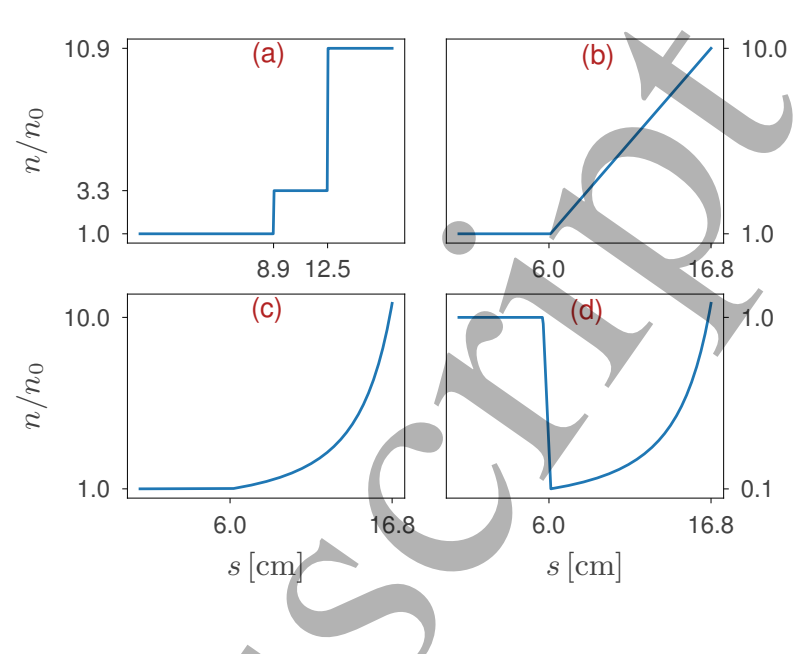

Figure 2. Plasma density profiles for the distinct types of varying plasma-density schemes: (a) multi-staged uniform density; (b) linearly increasing; (c) and (d) constant rate of plasma wavelength change.
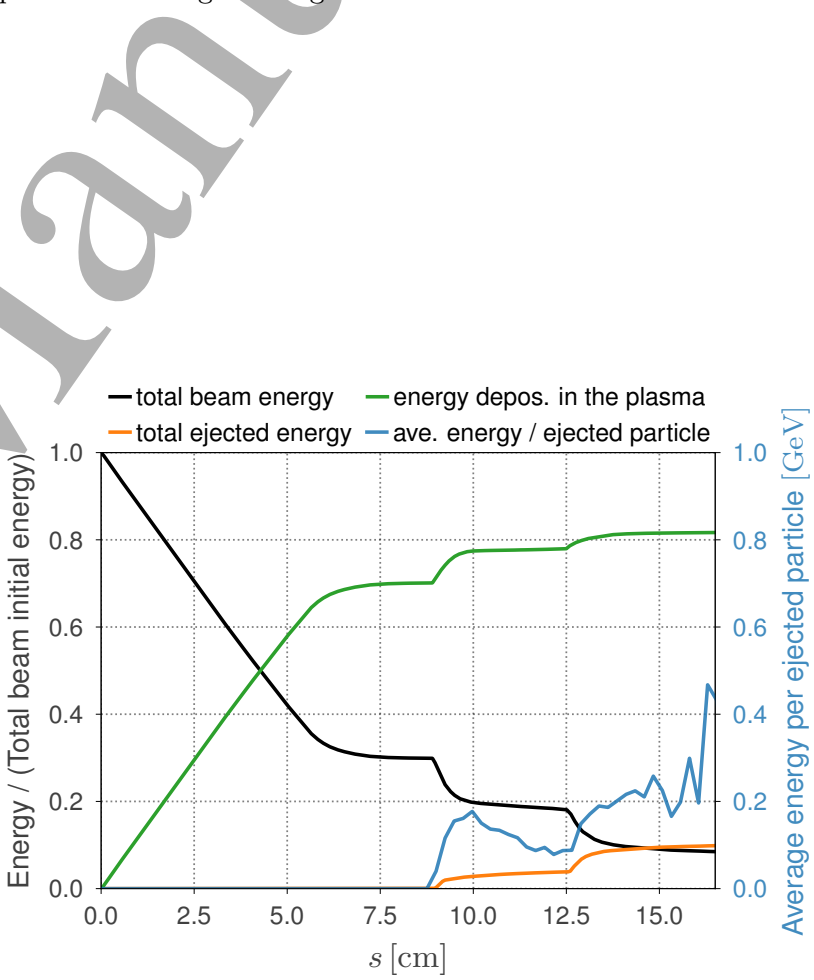

Figure 3. Multi-staged uniform density scheme. This plot depicts PIC simulation results for the plasma density profile shown in figure 2(a), with sharp density transitions from $n_{1}=$ $3.3 n_{0}$, at $s=8.9 \mathrm{~cm}$, and from $n_{2}=(3.3)^{2} n_{0}$, at $s=12.5$ $\mathrm{cm}$. Initially, the total beam energy (black line) is extracted in a linear fashion, being deposited into the plasma (green line). Due to re-acceleration of particles, the total beam energy loss saturates at $30 \%$ of its total initial energy $\left(U / U_{0} \simeq 0.3\right)$. Saturation is then "broken" by the first density transition, which causes the ejection of these particles. The total (cumulative) ejected energy (orange line), as well as the average energy per ejected particle (blue line) are also shown. A new saturation regime $\left(U / U_{0} \simeq 0.2\right)$ is broken by the second density transition. Although a total beam energy of $U / U_{0} \simeq 0.1$ is obtained, most of the extracted energy is now ejected, with a substantial increase in the average energy per ejected particle. 
a density change is set to place a transverse wakefield defocusing phase over this peak, to de-stabilize and disperse it by transversely ejecting its particles. If the re-acceleration peak has formed at the position of maximum longitudinal acceleration, then a plasma-density increase $n_{0} \rightarrow n_{1}$ will decrease the plasma wavelength and move the first defocusing region over the peak if $1<n_{1} / n_{0}<4$. Furthermore, half of this defocusing region is contained in the secondary decelerating region of the wakefield. Thus, by letting $2.25<n_{1} / n_{0}<4$, the re-acceleration peak will end up in a region of the wakefield that is simultaneously decelerating and defocusing. Based on the simulation results for a uniform plasma density profile (figure 1), a three-staged plasma beam dump, composed of consecutive density increases in this range, is suitable for the investigation. Propagation distances at which the density is increased are chosen to allow for the formation of sufficiently large reacceleration peaks. Thus, significant beam-energy reduction is attained when the re-acceleration peaks are defocused. Density changes of $n_{1} / n_{0}=n_{2} / n_{1}=3.3$, at $s=8.9 \mathrm{~cm}$ and $s=12.5 \mathrm{~cm}$, respectively, are chosen to provide large deceleration and weak defocusing, while avoiding the second focusing region at $n_{1} / n_{0}>4$. This plasma-density profile is shown in figure $2(\mathrm{a})$.

A PIC simulation was performed to evaluate the multi-staged uniform density scheme in a passive plasma beam dump. The length of the simulation window is chosen to be $\simeq 1.5 \lambda$, or $\simeq 52 \mu \mathrm{m}$, large enough to ensure that no particles leave the simulation in the longitudinal direction. While the width of the simulation window is $40 \sigma_{r}=56 \mu \mathrm{m}$ (from $-20 \sigma_{r}$ ) to $20 \sigma_{r}$ ), such that the transverse and longitudinal fields have decreased by a factor of 540 at the edge of the transverse simulation domain, ensuring that no appreciable re-acceleration occurs outside the window. The resulting total beam energy, as well âs the energy deposited in the plasma are shown in figure 3 , together with the total ejected energy from defocusing of the re-acceleration peaks. This is the cumulative energy of each particle that leaves the simulation window in the transverse direction. Moreover, the average energy per ejected particle (blue, right-sided vertical scale) is also shown in figure 3. These results show that around $90 \%$ of the total energy can/be extracted from the beam in a three-staged, $17 \mathrm{~cm}$ long plasma beam dump. Most of the energy extraction after saturation is due to electrons being ejected transversely at the points of density transition, with only minor increases in the energy deposited in the plasma. As expected, the first plasma density transition, $n_{1}=3.3 n_{0}$, at $s=8.9$ $\mathrm{cm}$, "breaks" the net energy loss saturation by placing the re-acceleration peak into a defocusing phase of the transverse wakefield. As a consequence, particles in this peak are transversely ejected. The total energy of the ejected particles rises from zero right after the density transition, with an average energy per ejected particle peaking at $\sim 200 \mathrm{MeV}$. After some propagation distance, the beam total energy loss is again saturated by the formation of a new re-acceleration peak. A new plasma density transition, $n_{2}=(3.3)^{2} n_{0}$, at $s=12.5 \mathrm{~cm}$, is used to eliminate this re-acceleration peak by ejecting its particles. However, figure 3 shows that the average energy per ejected particle reaches $\sim 400 \mathrm{MeV}$ after the second density transition. For a plasma vessel of a given size, the total power per unit area that the walls of the vessel would need to sustain could be estimated and compared to material data, in order to determine the feasibility of this scheme. The involvement of highly energetic electrons, however, means that this is not a trivial calculation to perform [35]. Since factors such as radiation emission, pair-production and ionization effects will need to be considered. In order to develop a beam dump with minimum radioactivation, a scheme which ejects particles of lower energy is desirable. The remainder of this paper attempts to address this issue.

\subsection{Linearly increasing density scheme}

Another approach to prevent saturation of the beam energy extraction is to set a continuously increasing density profile. As a first attempt, the linearly increasing density profile given by the following equation is a natural choice:

$$
n(s)= \begin{cases}n_{0} & , s<s_{\text {chirp }} \\ a\left(s-s_{\text {chirp }}\right)+n_{0} & , s \geq s_{\text {chirp }}\end{cases}
$$

where $s_{\text {chirp }}$ is the distance at which the first beam particles, i.e., those experiencing the higher amplitude decelerating wakefield, have reached non-relativistic velocities and start falling behind. As the density is linearly increased, the defocusing region will get progressively closer to the head of the bunch, where particles have higher energies. At some point, re-acceleration and subsequent defocusing of these particles will lead to their ejection. Hence, simulations have to be stopped before this point. For the adopted parameters, $s_{\text {chirp }}=6.1 \mathrm{~cm}$ is obtained from simulation results. Moreover, the plasma density profile is set to provide a 10-fold density increase over the propagation distance after $s_{\text {chirp}}$, i.e., $n(s=16.8 \mathrm{~cm})=10 n_{0}$. For longer distances, highly-relativistic particles (with $\gamma / \gamma_{0} \sim 1$ ) are ejected. This plasma density profile is shown in figure $2(\mathrm{~b})$.

PIC simulation results from figure 4 show that, although comparable energy extraction can be achieved in both multi-staged and linearly increasing density schemes, the latter shows a slightly increase in the total ejected energy, which implies a reduction 


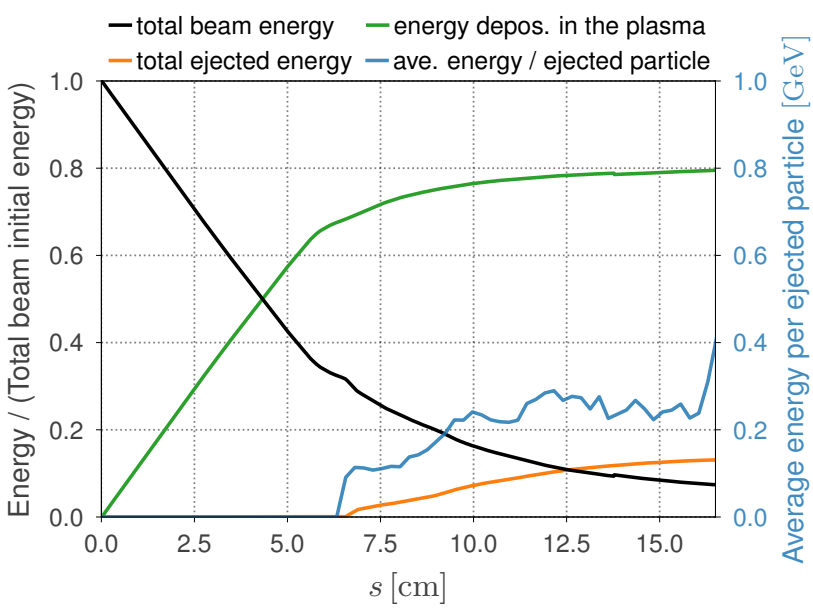

Figure 4. Linearly increasing density scheme. PIC simulation results for the plasma density profile shown in figure 2(b). The linearly increasing density prevents re-acceleration peak growth by defocusing its particles. This allows for further total beam energy extraction without saturation. Reduction of the defocusing-phase speed $\lambda^{\prime}$ as the beam propagates causes the average energy per ejected particle (blue line) to increase from $\sim 100 \mathrm{MeV}$ up to $\sim 300 \mathrm{MeV}$. Final total beam energy is $8 \%$ (black line), with $80 \%$ being deposited in the plasma (green line), and $12 \%$ being ejected (orange line).

of the energy deposited in the plasma. Regarding the average energy per ejected particle, although it is initially lower in the linearly increasing scheme around $100 \mathrm{MeV}$, in contrast to $200 \mathrm{MeV}$ in the multistaged density profile - it grows with a nearly-constant slope up to $300 \mathrm{MeV}$, at $s \simeq 12.5 \mathrm{~cm}$. After this point, the slope is greatly reduced. This behavior can be explained as follows. If the beam propagation is observed in the co-moving coordinate $\xi$, then the effect of continuously increasing (or decreasing) the plasma density can be appreciated as a continuous contraction (or expansion) of the plasma (and so the wakefield) wavelength. For a given varying density profile $n(s)$, the plasma wavelength is inversely proportional to the square root of the density, $\lambda=\kappa[n(s)]^{-1 / 2}$, with $\kappa \equiv$ $\left(\epsilon_{0} m_{e} c^{2} / e^{2}\right)^{1 / 2}>0$. Therefore, the speed at which this contraction (or expansion) takes place is given by the rate of wavelength change with respect to the distance, $\lambda^{\prime}=d \lambda / d s$. Moreover, $\lambda^{\prime}$ can be used to measure how fast a defocusing (or any other) wakefield phase is moving when the propagation is observed with the co-moving coordinate $\xi$. In particular, for the linearly increasing density profile from equation (2), the speed $\lambda^{\prime}$ is given by the following expression,

$$
\lambda^{\prime}(s)=-\frac{c_{1}}{\left[c_{2}+\left(s-s_{\text {chirp }}\right)\right]^{3 / 2}}, s \geq s_{\text {chirp }}
$$

where $c_{1}=(\kappa / 2) a^{-1 / 2}>0$, and $c_{2}=n_{0} / a>0$. The negative sign of $\lambda^{\prime}$ indicates that the wavelength is contracting, as expected for an increasing density

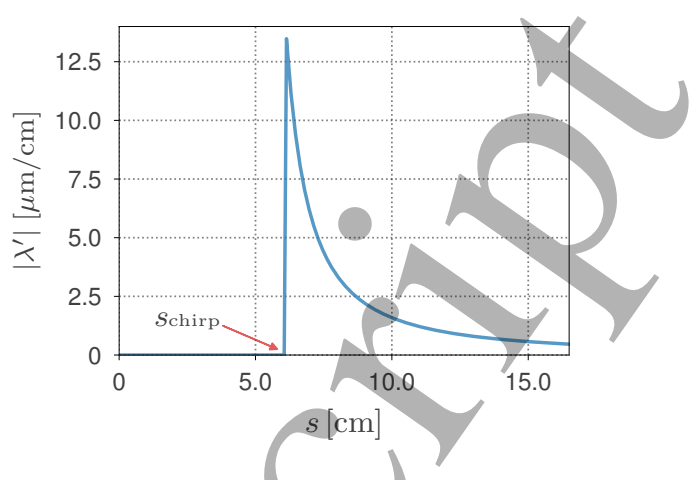

Figure 5. Speed at which the defocusing phase of the wake moves towards the head of the bunch in the linearly increasing density scheme. Values obtained for the set of parameters under investigation.

profile, and $\left|\lambda^{\prime}\right|$ gives the speed of the contraction. Figure 5 exhibits $\left|\lambda^{\prime}\right|$ in $\mu \mathrm{m} / \mathrm{cm}$ for the current set of parameters. The chosen unit gives the distance that the defocusing region moves closer to the beam per centimetre of beam propagation, when observed from the co-moving coordinate $\xi$. Soon after $s_{\text {chirp }}=6.1$ cm, the defocusing phase of the wakefield moves at $\lambda^{\prime} \simeq-14 \mu \mathrm{m} / \mathrm{cm}$, which is the maximum value in the linearly increasing scheme. However, this speed falls quickly as the beam propagates. For example, at $s=10 \mathrm{~cm}, \lambda^{\prime}$ already shows an 9-fold reduction $\left(\lambda^{\prime} \underline{\simeq}-1.6 \mu \mathrm{m} / \mathrm{cm}\right)$. Therefore, the higher the density in the linearly increasing scheme, the lower the speed of the defocusing wake phase, $\lambda^{\prime}$. For this reason, as the beam propagates in this scheme, particles stay for longer times in the accelerating phase prior to defocusing, hence being ejected with higher energies. For longer distances, the speed tends asymptotically to zero, allowing decelerated particles to stay in the accelerating phase, giving rise to a secondary peak.

\subsection{Constant rate of wavelength change}

An alternative to solve the issue observed in the linearly increasing density scheme, in which the rate of wavelength change $\lambda^{\prime}$ decreases for higher densities, is to derive a density profile that provides a constant rate of wavelength change. Since $\lambda \propto 1 /[n(s)]^{-1 / 2}$,

$$
\frac{\mathrm{d}^{2} \lambda}{\mathrm{d} s^{2}} \propto\left[\frac{n^{\prime \prime}}{n^{3 / 2}}-\frac{3}{2} \frac{\left(n^{\prime}\right)^{2}}{n^{5 / 2}}\right]
$$

By setting $\mathrm{d}^{2} \lambda / \mathrm{d} s^{2}=\lambda^{\prime \prime}=0$ in equation (4), a second-order, non-linear ordinary differential equation is obtained for the plasma density, which satisfies

$$
n(s)=\left\{\begin{array}{ll}
n_{0} & , s<s_{\text {chirp }} \\
\frac{c_{3}}{\left(c_{4}-s\right)^{2}} & , s_{\text {chirp }} \leq s<c_{4}
\end{array},\right.
$$


where the constants $c_{3}>0$ and $c_{4}>0$ are determined from the boundary conditions. For the plasma density profile described by equation (5), the rate of plasma wavelength change and thus the speed of the wakefield defocusing-phase is constant when observed from the co-moving coordinate $\xi$, and given by $\lambda^{\prime}=-\kappa / \sqrt{c_{3}}$. In

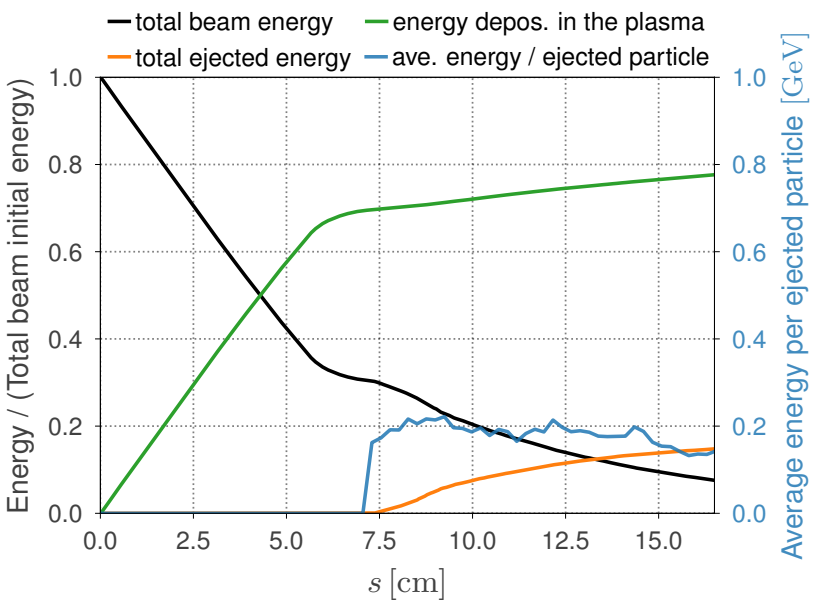

Figure 6. Constant rate of wavelength change scheme. PIC simulation results for the plasma density profile shown in figure $2(\mathrm{c})$. As the beam propagates, particles are then ejected with a constant defocusing-phase speed $\left(\lambda^{\prime} \simeq-2.2 \mu \mathrm{m} / \mathrm{cm}\right)$. The average energy per ejected particle (blue line) is nearly constant at $\sim 200 \mathrm{MeV}$. Final total beam energy is $8 \%$ (black line), with $77 \%$ being deposited in the plasma (green line), and $15 \%$ being ejected (orange line).

order to compare this density profile with the linearly increasing case from equation $(2), n\left(s_{\text {chirp }}=6.1 \mathrm{~cm}\right)=$ $n_{0}$ and $n(s=16.6 \mathrm{~cm})=10 n_{0}$ are chosen as boundary conditions, resulting in the density profile shown in figure 2(c). This yields a speed of $\lambda^{\prime} \simeq-2.2 \mu \mathrm{m} / \mathrm{cm}$ all along the beam propagation after $s_{\text {chirp. }}$. The effect of having defocusing at a constant speed can be seen in figure 6 , with PIC simulation results showing an almost-constant average energy per ejected particle of $\sim 200 \mathrm{MeV}$. In this case, the final beam total energy is lower than $10 \%\left(U / U_{0} / \simeq 0.08\right)$, with approximately $77 \%$ being deposited in the plasma, and $15 \%$ being ejected. Since a faster speed $\lambda^{\prime}$ would cause earlier defocusing of the re-acceleration peak, this would cause particles to be ejected with lower energies. Therefore, a natural approach to reduce the average energy per ejected particle would be to play with the boundary conditions when solving equation (5), aiming for a higher value of the speed $\lambda^{\prime}$. However, this cannot be achieved by maintaining the boundary condition in $s_{\text {chirp }}, n\left(s_{\text {chirp }}=6.1 \mathrm{~cm}\right)=n_{0}$, and choosing a higher density increase in the second one, at $s=16.6$ $\mathrm{cm}$. For example, the choice of a 100-fold density increase, $n(s=16.6 \mathrm{~cm})=100 n_{0}$, which is 10 -times higher than the previous choice, results in a speed $\lambda^{\prime} \simeq$ $-2.9 \mu \mathrm{m} / \mathrm{cm}$. This value is just slightly higher than the $-2.2 \mu \mathrm{m} / \mathrm{cm}$ previously obtained. Although an effective increase of $\lambda^{\prime}$ could be obtained by shortening the length over which the density increases, this would cause premature defocusing of high-energy particles located closer to the head of the bunch.

One solution to achieve a higher speed $\lambda^{\prime}$ while maintaining the same 10-fold density-increase and propagation distance $s_{\text {chirp }}=6.1 \mathrm{~cm} \rightarrow 16.6 \mathrm{~cm}$ is to set a 10 -times density-drop $\left(n_{0} \rightarrow 0.1 n_{0}\right)$ at $s_{\text {chirp }}$. Figure 2(d) shows the plasma density profile obtained by solving equation (5) with $n\left(s_{\text {chirp }}\right)=0.1 n_{0}$ and $n(s=16.6 \mathrm{~cm})=n_{0}$ as boundary conditions. Because a sudden density-drop instantly lowers the amplitude of the focusing wakefield, it will cause ejection of highenergy particles across the head of the bunch. To mitigate this effect, a short, 5-millimeter-long density down-ramp was added. This density profile now provides a speed of $\lambda^{\prime}=-6.9 \mu \mathrm{m} / \mathrm{cm}$, a value 3.2 times higher than the one obtained in the previous case (with no density drop). PIC simulation results for the plasma

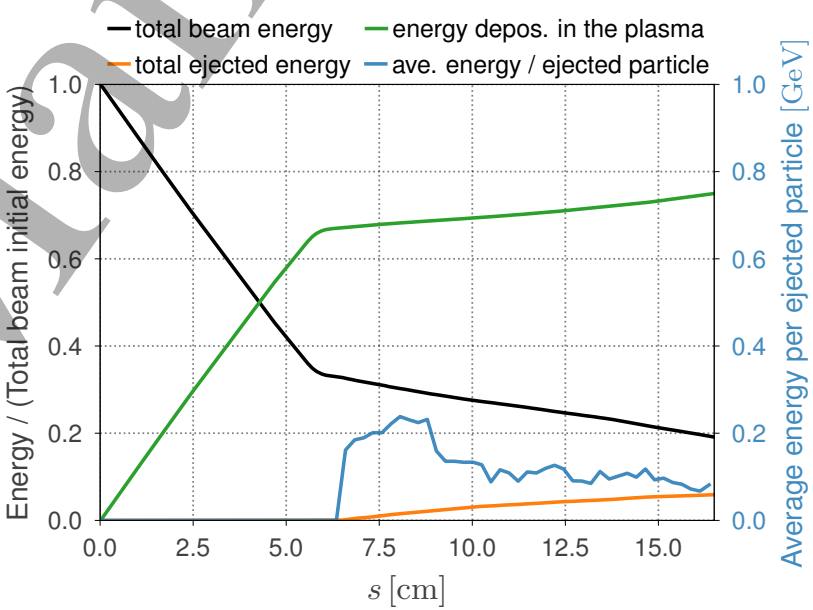

Figure 7. Constant rate of wavelength change scheme (with density drop). PIC simulation results for the plasma density profile shown in figure $2(\mathrm{~d})$. A $5 \mathrm{~mm}$ long down-ramp drops the density from $n_{0}$ down to $0.1 n_{0}$ at $s_{\text {chirp }}=6.1 \mathrm{~cm}$. Particles are then ejected with a constant defocusing-phase speed of $\lambda^{\prime} \simeq$ $-6.9 \mu \mathrm{m} / \mathrm{cm}$, which is 3.2 times higher than the constant speed previously obtained $\left(\lambda^{\prime} \simeq-2.2 \mu \mathrm{m} / \mathrm{cm}\right)$. Due to the sudden density drop, high-energy particles at the head of the beam are ejected, causing a localized increase in the average energy per ejected particle (blue line) that peaks at $220 \mathrm{MeV}$. After that, the average energy per ejected particle stabilizes around $100 \mathrm{MeV}$. Final total beam energy is $19 \%$ (black line), with $75 \%$ being deposited in the plasma (green line), and $6 \%$ being ejected (orange line).

density profile from figure 2(d) are displayed in figure 7. In this figure, the effect of having a density drop can be observed in the average energy per ejected particle. Here, an initial localized increase, peaking at $220 \mathrm{MeV}$ at $8 \mathrm{~cm}$, is caused by ejection of a small number of highenergy electrons from the head of the bunch, right after the density drop. Although the adopted down-ramp 


\section{Tailored plasma-density profiles}

certainly helped, its simple shape was not enough to eliminate undesired effects caused by the density drop. By adopting some of the specific density gradients used in experiments to mitigate beam effects of this nature when exiting a plasma $[36,37]$, it might be possible to minimize the initial increase in the average energy per ejected particle. Once this effect has ceased, particles are defocused with an average energy of $\sim 100 \mathrm{MeV}$, which is maintained up to the end of the simulation. This value, which is $50 \%$ lower than the previous one, is in agreement with the idea that a defocusing phase moving at a higher speed $\lambda^{\prime}$ provides earlier defocusing, ejecting particles with lower energies. Despite the higher final total beam energy $(19 \%$, in contrast to $8 \%$ in the previous case), the total energy deposited in the plasma in both cases is very similar $(75 \%$ here, and $77 \%$ in figure 6). The main difference is in the total ejected energy, which changed from $15 \%$ in the previous case to only $6 \%$ in this simulation. This reduction is explained by the lower average energy per ejected particle.

\section{Summary and conclusions}

In this work, tailored plasma-density profiles have been used to overcome beam energy loss saturation observed in passive plasma beam dumps with uniform density profiles. Longitudinal plasma-density shaping is set to decrease the wavelength of the beam-driven wakefield, shifting its defocusing phase towards reaccelerated beam particles in order to have them transversely ejected. It is shown that $\lambda^{\prime}$, the rate at which the wavelength decreases with respect to the propagation distance, plays a significant role in the energy of ejected particles. Since $\lambda^{\prime}$ denotes the speed at which the wakefield defocusing-phase moves when the beam propagation is observed with the comoving coordinate $\xi$, higher values of $\lambda^{\prime}$ lead to earlier defocusing, reducing the time that decelerated beam particles experience re-acceleration. Hence, particles are ejected with lower energies.

Three distinct density-varying schemes were investigated. In the multi-staged uniform density scheme, approximately $90 \%$ of the total beam energy is extracted, with $80 \%$ being deposited into the plasma. However, particles with an average energy above 400 $\mathrm{MeV}$ are ejected. In the linearly increasing density scheme, similar results are obtained (figure 4) for the beam energy extraction, and the energy deposited into the plasma. Regarding the average energy of ejected particles, it increases from $\sim 100 \mathrm{MeV}$ up to $\sim 300$ $\mathrm{MeV}$ as the beam propagates, beyond which point higher-energy particles are ejected. This behavior can be explained by the reduction of the defocusingphase speed $\lambda^{\prime}$ in this profile (figure 5). Lower, quasiuniform average energies are observed for the ejected particles in both plasma density profiles derived to provide constant speed $\lambda^{\prime}$. The first case (figure 6) shows particles being ejected with an average energy of $\sim 200 \mathrm{MeV}$ all along the beam propagation. Total beam energy loss and energy deposited in the plasma are comparable with previous schemes. In the second case, a density-drop allows for tripling the speed $\lambda^{\prime}$. PIC simulation results (figure 7) show an initial localized increase in the average energy of ejected particles, peaking at $\sim 220 \mathrm{MeV}$, caused by the nonoptimized down-ramp profile adopted for the density drop. After the initial increase, the average energy per ejected particle stabilizes around $100 \mathrm{MeV}$, showing a reduction of $50 \%$ in comparison to the previous case. This validates the idea that a higher speed $\lambda^{\prime}$ provides earlier defocusing, ejecting particles with lower energies. For the total beam energy, a lower energy extraction $(81 \%)$ is observed for this profile.

The proposed density-varying schemes have prevented energy loss saturation, enabling beam energy extraction up to $\sim 90 \%$ for most of the investigated cases. The obtained results reduce the existing gap between passive and active plasma beam dump schemes. However, further investigation is required to lower the energy of ejected particles. Values under $10 \mathrm{MeV}$ are desired, as they would not lead to appreciable levels of radioactivation [18]. In this way, future works will be conducted to define the optimal behavior of the speed $\lambda^{\prime}$ based in the dynamics of decelerated particles, aiming for particle ejection at lower energies.

Experimental implementation of the proposed plasma-density profiles would certainly be challenging at the present time. Serial combination of homogeneous plasma cells and/or gas jets could be an alternative to implement quasi-sharp density transitions and down-ramps. However, dealing with interfaces between serial stages, and properly ionizing the plasma for such setups are both non-trivial tasks. Implementation of tapered plasma channels [38] has been discussed in the context of electron acceleration, and a factor of 2 was obtained in the on-axis plasma density over a $2 \mathrm{~cm}$ long capillary. In the AWAKE experiment [12], a linear density variation of $20 \%$ over a 10 -meter long plasma was implemented and controlled at a $1 \%$ level. This was achieved by heating the plasma source extremities at distinct temperatures. Existing results [39] show nonlinearly increasing profiles which lightly resemble figure 2 (c). In such profiles, a factor of $\sim 2.5$ was obtained in the density of a $2 \mathrm{~cm}$ long plasma. The mentioned results show that tailored plasma-density profiles are an active subject of research. Therefore, longer plasmas, with higher density-change factors, might be available in the near future, allowing for experimental verification of the theoretical results presented here. 


\section{Acknowledgments}

This work was supported by the Cockcroft Institute Core Grant and the STFC. The authors acknowledge computing resources provided by STFC Scientific Computing Department's SCARF cluster, and the University of Manchester's CSF3 cluster. Moreover, the authors thank Rémi Lehe by his support regarding the use of FBPIC.

\section{References}

[1] Tajima T and Dawson J M 1979 Phys.Rev.Lett. $43267-270$

[2] Esarey E, Schroeder C B and Leemans W P 2009 Rev. Mod. Phys. 81 1229-1285

[3] Gonsalves A J, Nakamura K, Daniels J, Benedetti C, Pieronek C, de Raadt T C H, Steinke S, Bin J H, Bulanov S S, van Tilborg J, Geddes C G R, Schroeder C B, Toth C, Esarey E, Swanson K, Fan-Chiang L, Bagdasarov G, Bobrova N, Gasilov V, Korn G, Sasorov P and Leemans W P 2019 Physical Review Letters 122084801

[4] Steinke S, van Tilborg J, Benedetti C, Geddes C G R, Schroeder C B, Daniels J, Swanson K K, Gonsalves A J, Nakamura K, Matlis N H, Shaw B H, Esarey E and Leemans W P 2016 Nature $5301-9$

[5] Schroeder C B, Esarey E, Geddes C G R, Benedetti C and Leemans W P 2010 PRST Accel. Beams 13101301

[6] Nakajima K 2018 Light: Science \&s Applications $1-3$

[7] Chen P, Dawson J, Huff R and Katsouleas T 1985 Phys.Rev.Lett. 54 693-696

[8] Blumenfeld I, Clayton C E, Decker F J, Hogan M J, Huang C, Ischebeck R, Iverson R, Joshi C, Katsouleas T and Kirby N 2007 Nature 445 741744

[9] Adli E, Ahuja A, Apsimon O, Apsimon R, Bachmann A M, Barrientos D, Batsch F, Bauche J, Olsen V K B, Bernardini M, Bohl T, Bracco C, Braunmüller F, Burt G, Butteńschön B, Caldwell A, Cascella M, Chappell J, Chevallay E, Chung M, Cooke D, Damerau H, Deacon L, Deubner L H, Dexter A, Doebert S, Farmer J, Fedosseev V N, Fiorito R, Fonseca R A, Friebel F, Garolfi L, Gessner S, Gorgisyan I, Gorn A A, Granados E, Grulke O, Gschwendtner E, Hansen J, Helm A, Henderson J R, Hüther M, Ibison M, Jensen L, Jolly S, Keeble F, Kim S Y, Kraus F, Li Y, Liu S, Lopes N, Lotov K V, Brun L M, Martyanov M, Mazzoni S, Godoy D M, Minakov V A, Mitchell J, Molendijk J C, Moody J T, Moreira M, Muggli
P, Oz E, Pasquino C, Pardons A, Asmus F P, Pepitone K, Perera A, Petrenko A, Pitman S, Pukhov A, Rey S, Rieger K, RUHL H, Schmidt J S, Shalimova I A, Sherwood P, Silva L O, Soby L, Sosedkin A P, Speroni R, Spitsyyn R I, Tuev P V, Turner M, Velotti F, Verra L, Verzilov V A, Vieira J, Welsch C P, Williamson B, Wing M, Woolley B and Xia G 2018 Nature 1-6

[10] D'Arcy R, Aschikhin A, Bohlen S, Boyle G, Brümmer T, Chappell J, Diederichs S, Foster B, Garland M J, Goldberg L, Gonzalez P, Karstensen S, Knetsch A, Kuang P, Libov V, Ludwig K, Martinez de la Ossa A, Marutzky F, Meisel M, Mehrling T J, Niknejadi P, Poder K, Pourmoussavi P, Quast M, Röckemann J H, Schaper L, Schmidt B, Schröder S, Schwinkendorf J P, Sheeran B, Tauscher G, Wesch S, Wing M, Winkler P, Zeng M and Osterhoff J 2019 Philosophical Transactions of the Royal Society A: Mathematical, Physical and Engineering Sciences 377 20180392-16

[11] Joshi C, Adli E, An W, Clayton C E, Corde S, Gessner S, Hogan M J, Litos M, Lu W, Marsh K A, Mori W B, Vafaei-Najafabadi N, O'Shea B, Xu X, White G and Yakimenko V 2018 Plasma Phys. Control. Fusion 60 034001-15

[12] Gschwendtner E, Turner M, Adli E, Ahuja A, Apsimon O, Apsimon R, Bachmann A M, Batsch F, Bracco C, Braunmüller F, Burger S, Burt G, Buttenschön B, Caldwell A, Chappell J, Chevallay E, Chung M, Cooke D, Damerau H, Deubner L H, Dexter A, Doebert S, Farmer J, Fedosseev V N, Fiorito R, Fonseca R A, Friebel F, Garolfi L, Gessner S, Goddard B, Gorgisyan I, Gorn A A, Granados E, Grulke O, Hartin A, Helm A, Henderson J R, Hüther M, Ibison M, Jolly S, Keeble F, Kelisani M D, Kim S Y, Kraus F, Krupa M, Lefevre T, Li Y, Liu S, Lopes N, Lotov K V, Martyanov M, Mazzoni S, Minakov V A, Molendijk J C, Moody J T, Moreira M, Muggli P, Panuganti H, Pardons A, Peña Asmus F, Perera A, Petrenko A, Pukhov A, Rey S, Sherwood P, Silva L O, Sosedkin A P, Tuev P V, Velotti F, Verra L, Verzilov V A, Vieira J, Welsch C P, Wendt M, Williamson B, Wing M, Woolley B, Xia G and The AWAKE Collaboration 2019 Philosophical Transactions of the Royal Society A: Mathematical, Physical and Engineering Sciences 377 20180418-9

[13] Ferry S and Karantzoulis E 2010 Proceedings of PAC'09 2887-2889

[14] Sola E L, Aberle O, Avigni P, Bianchi L, Busom J, Calviani M, Casolino M, Espadanal J P C, Fraser M A, Girod S, Goddard B, Grenier D, Guinchard 
M, C Heßler C H, Fiastre R I, Jacobsson R, Lamont M, Rolo A O, Riffaud B, Romagnoli G, CERN L Z, Geneva and Switzerland 2018 Proceedings of IPAC'18 2604-2607

[15] Schumann D, Neuhausen J, Eikenberg J, Rüthi M, Wohlmuther M, Kubik P W, Synal H A, Alfimov M V, Korschinek G, Rugel G and Faestermann T 2009 Radiochimica Acta International journal for chemical aspects of nuclear science and technology 97 123-131

[16] Leuschner A and Tesch K 1998 Internal Report DESY D3-92

[17] Walker R P and Bartolini R e a 2010 accelconf.web.cern.ch

[18] Wu H C, Tajima T, Habs D, Chao A W and Meyer-ter Vehn J 2010 PRST Accel. Beams 13 101303

[19] Hanahoe K, Xia G, Islam M, Li Y, Mete-Apsimon Ö, Hidding B and Smith J 2017 Physics of Plasmas (1994-present) 24 023120-7

[20] Bonatto A, Schroeder C B, Vay J L, Geddes C G R, Benedetti C, Esarey E and Leemans W P 2015 Phys. Plasmas 22 083106-083113

[21] Chou S W Investigation of electron acceleration and deceleration in plasmas Ph.D. thesis Fakultaet fuer Physik, Muenchen University, Germany

[22] Rykovanov S G, Geddes C G R, Vay J L, Schroeder C B, Esarey E and Leemans W P 2014 Journal of Physics B: Atomic, Molecular and Optical Physics 47 1-22

[23] Geddes C G, Steinke S, van Tilborg J, Shaw B, Matlis N, Gonsalves A J, Nakamura K, Huijts J, Mittelberger D, Daniels J, Toth C, Vay J L, Bonatto A, Rykovanov S, Schroeder C, Benedetti C, Esarey E and Leemans W P 2015 Frontiers in Optics 2015 (2015), paper FM2A.3

[24] Shpakov V, Anania M P, Bellaveglia M, Biagioni A, Bisesto F, Cardelli F, Cesarini M, Chiadroni E, Cianchi A, Costa G, Croia M, Del Dotto A, Di Giovenale D, Diomede M, Ferrario M, Filippi F, Giribono A, Lollo V, Marongiu M, Martinelli V, Mostacci A, Piersanti L, Di Pirro G, Pompili R, Romeo S, Scifo J, Vaccarezza C, Villa F and Zigler A 2019 Phys.Rev.Lett. 122114801

[25] D'Arcy R, Wesch S, Aschikhin A, Bohlen S, Behrens C, Garland M J, Goldberg L, Gonzalez P, Knetsch A, Libov V, de la Ossa A M, Meisel M, Mehrling T J, Niknejadi P, Poder K, Röckemann J H, Schaper L, Schmidt B, Schröder S, Palmer C, Schwinkendorf J P, Sheeran B, Streeter M J V, Tauscher G, Wacker V and Osterhoff J 2019 Phys.Rev.Lett. 122034801
[26] Adli E, Lindstrøm C A, Allen J, Clarke C I, Frederico J, Gessner S J, Green S Z, Hogan M J, Litos M D, O'Shea B, Yakimenko V, An W, Clayton C E, Marsh K A, Mori W B, Joshi C, Vafaei-Najafabadi N, Corde S and Lu W 2016 New J. Phys 18 103013-11

[27] Nunes R, Bonatto A and Xia G 2019 Proceedings of IPAC'19 3581-3583

[28] Bonatto A, Nunes R and Xia G 2019 Proceedings of IPAC'19 3584-3586

[29] Lu W, Huang C, Zhou M M, Mori W B and Katsouleas T 2005 Phys Plasmas 12063101

[30] Chou S, Xu J, Khrennikov K, Cardenas D E, Wenz J, Heigoldt M, Hofmann L, Veisz L and Karsch S 2016 Physical Review Letters 117144801

[31] Li X, Mosnier A and Nghiem P A P 2018 Nuclear Inst. and Methods in Physics Research, A 1-5

[32] Lehe R, Kirchen M, Andriyash I A, Godfrey B B and Vay J L 2016 Computer Physics Communications 203 66-82

[33] Jalas S, Dornmair I, Lehe R, Vincenti H, Vay J/L, Kirchen M and Maier A R 2017 Physics of Plasmas 24 033115-8

[34] Godfrey B B 1974 Journal of Computational Physics 15 504-521

[35] Fernandez-Hernando J L and Angal-Kalinin D 2010 Proceedings of IPAC'10 1805-1807

[36] Ariniello R, Doss C E, Hunt-Stone K, Cary J R and Litos M D 2019 Phys. Rev. Accel. Beams 22 041304

[37] Li X, Chancé A and Nghiem P A P 2019 Phys. Rev. Accel. Beams 22021304

[38] Sprangle P, Hafizi B, Peñano J R, Hubbard R F, Ting A, Moore C I, Gordon D F, Zigler A, Kaganovich D and Antonsen T M 2001 Phys. Rev. E 63(5) 056405

[39] Filippi F, Anania M P, Biagioni A, Brentegani E, Chiadroni E, Cianchi A, Deng A, Ferrario M, Pompili R, Rosenzweig J and Zigler A 2018 Nuclear Inst. and Methods in Physics Research, A 909 339-342 